\title{
The Role of Biofilms in Pathology of the Ocular Surface
}

\author{
Jesse Pelletier \\ Ocean Ophthalmology Group, Miami, FL, USA
}

DOI: https://doi.org/10.17925/OPHT.2021.15.1.12

$\mathrm{N}$ ewer classification schemes with respect to ocular surface disease (OSD) highlight the complexity of pathology and illuminate areas of potential scientific interest. One critical aspect of blepharitis is the formation of bacterial biofilms. Biofilms are composed of bacterial communities ensconced within extracellular matrices. These specialized ecosystems allow for enhanced communication and, ultimately, the ability to survive in harsh environments. Their role in the development of OSD, including dry eye and blepharitis, is only now being elucidated. Therapeutic strategies to eliminate ocular biofilms include the use of mechanical exfoliation, antibiotics and compounds containing hypochlorous acid. Novel formulations of povidone-iodine may also be useful in combatting biofilms and ultimately restoring ocular surface homeostasis.

\section{Keywords}

Biofilm, ocular surface disease, dry eye, blepharitis, povidone-iodine

Disclosure: Jesse Pelletier is a co-founder and chief medical officer of Veloce BioPharma.

Review process: Double-blind peer review.

Compliance with ethics: This study is an opinion piece and does not report on new clinical data, or any studies with human or animal subjects performed by the author.

Authorship: The named author meets the International Committee of Medical Journal Editors (ICMJE) criteria for authorship of this manuscript, takes responsibility for the integrity of the work as a whole, and has given final approval for the version to be published.

Access: This article is freely accessible at touchOPHTHALMOLOGY.com @ Touch Medical Media 2021.

Received: 13 April 2021

Accepted: 25 June 2021

Published online: 14 July 2021

Citation: touchREVIEWS in Ophthalmology. 2021; 15(1):12-4

Corresponding author: Jesse Pelletier, Ocean Ophthalmology Group, 1400 NE Miami, Gardens Drive, Ste, 203, North Miami Beach, FL, 33132, USA E: jesse.pelletier@oceanophthalmology.com

Support: No funding was received in

the publication of this article.
Over the past few years, we have witnessed an increasing awareness of the importance of treating ocular surface disease (OSD). Diagnoses such as dry eye and blepharitis, which were historically under-appreciated, now command attention from the ophthalmologist and investment from the ocular pharmaceutical industry. Proper treatment of the ocular surface is paramount to the health of our patients because the morbidity is real and the scope of the problem is large. Dry eye affects 30 million or more people in the USA, and up to half of patients presenting to eye-care specialists may suffer from blepharitis. ${ }^{1,2}$ In support of this, we have witnessed the commitment of big pharma and biotech alike to these causes, ensuring that both our armamentarium and its deployment will look distinctly different in the future. Moreover, the support of academia in the publication of treatment algorithms such as DEWS II and CEDARS has underscored the complexity of these pathologies and offered guidance to the treating physician. ${ }^{3,4}$ As we begin to unravel the nature of OSD and specifically blepharitis, novel therapeutic targets come into focus. One such target at the forefront of antimicrobial research is the bacterial biofilm. Recent interest in a "biofilm theory of dry eye disease ${ }^{\prime \prime}$ as the natural sequelae of decades of chronic blepharitis, ${ }^{6}$ highlights the attention the role of biofilm in OSD has garnered.

Biofilms have been around since the beginning of time. They are well-documented components of primordial slime and deep-sea hyperthermic vents. Elucidating their role in humans, and especially ocular disease, has come at slow pace. When understanding the biofilm, we must acknowledge that bacteria behave differently under certain conditions. A planktonic or solitary bacterium is distinct from a sessile network of other bacteria as components in a biofilm. Biofilms comprise an extracellular polymeric substance matrix secreted by bacteria on a living or abiotic surface, which facilitates the development of a specialized community capable of communication, pooling of

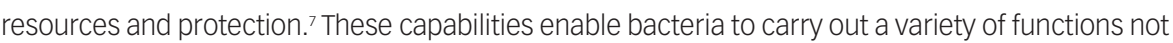
accessible to planktonic bacterial systems, which can lead to the overwhelming of host defences and infection. It is in this networked microbiological milieu that the answer to some difficult questions about how antibiotic resistance develops and how otherwise benign commensal microorganisms become pathogenic may lie.

To better understand the biofilm, it is crucial to examine its components. Its formation is known to occur in distinct stages. The first involves attachment of the planktonic bacteria to a surface. This is followed by adhesion, the formation of microcolonies, maturation and eventual detachment of the cells. ${ }^{8}$ The ultrastructure of a biofilm is understood through imaging with techniques such as confocal or electron microscopy. It contains polymeric substances, such as polysaccharides, nucleic acids and proteins, which are built up over a substrate and result in a three-dimensional biomass. ${ }^{9}$ Within this structure, a delicate homeostasis is achieved among similar or diverse species of bacteria. The organization of the matrix provides for water channels and varying gradients of nutrients, oxygen and $\mathrm{pH}$, which all contribute to the formation of heterogeneous microcolonies and phenotypes within the biofilm. These microcolonies may take certain shapes, such as pillar or mushroom-shaped structures. Those in close proximity to the nutrients may be more metabolically active, while others that are more deeply embedded may lie in a state of 
dormancy. This now fully-organized secretion is primed to facilitate cellto-cell interaction and manipulate gene expression.

At first look, the ocular surface does not seem like an environment hospitable to host commensal bacteria. It is a harsh and austere place for colonization. Numerous deterrents are present, including mucins, secreted by goblet cells to act as anti-adhesives; proteins, such as lactoferrin, lysozyme and defensins, to inhibit the bacterial growth cycle; the constant flow of tears; and the mechanical action of the eye during the blinking process. ${ }^{10}$ Nonetheless, we are aware of several bacteria able to surmount these obstacles. They include species of Staphylococcus, Streptococcus, Corynebacterium and Propionibacterium.11,12 When probing for host weakness, microorganisms are likely to find certain anatomical locations on the ocular surface more desirable than others, such as the eyelid margin. We know this particular area of the body is not cleaned frequently through normal hygiene, and the sebaceous/meibomian glands provide nutritious secretions to propagate bacterial growth.

It has traditionally been taught that biofilms in ophthalmology are responsible for a wide variety of pathologies, including endophthalmitis, keratitis, lacrimal system disease and periorbital infections. ${ }^{13}$ Most of these manifestations are thought to develop from the introduction of foreign materials, such as intraocular lenses, contact lenses, scleral buckles, keratoprostheses, orbital implants and punctal plugs, which may provide a charged surface on which bacteria initially attach. ${ }^{14,15}$ There is, however, a growing body of evidence that the eye itself, specifically the lid margin, is subject to the development of this biofilm naturally and without the prerequisite of foreign material introduction. Biofilms are typically found at the junction of mucous membranes, and it has been suggested that biofilm builds up year after year, until the bioburden results in vast over-colonization and eventual pathology in the form of blepharitis and even dry eye. ${ }^{6}$ It has also been reported that biofilms of the eyelids and eyelashes may specifically contribute to the difficulty of treating infectious blepharitis. ${ }^{16}$ Microorganisms ensconced in a biofilm matrix may be up to 1,000 times more resistant to antibiotics. ${ }^{17}$

The further examination of bacterial species, such as Staphylococcus spp., reveals prerequisites of gene expression necessary for biofilm production. For example, strains of S. epidermidis and coagulasenegative Staphylococci contain the ica operon responsible for the production of a homoglycan called polysaccharide intercellular adhesin. ${ }^{18}$ This molecule is responsible for the critical process of initial bacterial attachment, along with biocide resistance and inhibition of neutrophil-dependent killing. Once this first stage of biofilm formation is complete, the process of quorum sensing is a cornerstone to intercellular communication. Homoserine lactones along with potassium-mediated ionic signals aid in this cell-to-cell signalling and may be responsible for an eventual change in gene expression. With respect to virulence, it is generally accepted that coagulase-negative Staphylococci encode fewer putative factors than S. aureus. ${ }^{19}$ Operons encoding for chemical signalling and expression of virulence factors, such as enterotoxins, leucocidins, alpha-toxin and protein A, play an important role in the development of $S$. aureus pathology.

The question of how to best combat robust biofilms responsible for human disease remains in play. It is prevailing thought that preventing the initial step of adhesion or destroying the mature biofilm represent good options. This can be accomplished by modifying surface topology of an implanted device or utilizing biocidal molecules. Some of the most well-regarded biofilm-destroying agents include small molecules, silver ions, nanoparticles, antiseptics, detergents, naturally occurring plant compounds and certain antibiotics. In vitro assays have also been developed, such as the Calgary Biofilm Device (MBEC Biofilms Technology Ltd., Calgary Alberta, Canada), microtitre plates, flow cell model, or suspended substratum reactor, which may assist in rapidly determining anti-biofilm susceptibilities. ${ }^{20}$

In the field of ophthalmology, promising research has been conducted with azithromycin ophthalmic solution (Azasite ${ }^{\circledR}$; Insite Vision, Inc., Alameda, CA, USA), the BlephEX ${ }^{\circledast}$ device (Scope Ophthalmics, Crawley, UK), hypochlorous acid-containing compounds, and most recently, novel formulations of dilute povidone-iodine. ,5, $, 21,22$ Although mechanical-type debridement at the lid margin to improve meibomian gland function and reduce dry eye symptoms is a good start, ${ }^{23}$ patients may be treated more comprehensively through chemical means that not only address the biofilm but also reduce oxidative stress and provide neurostimulatory effects. Novel formulations of dilute povidone-iodine offer this possibility and have demonstrated both in vitro effectiveness against established biofilms of multidrug-resistant $S$. aureus, Klebsiella pneumoniae, Pseudomonas aeruginosa and Candida albicans, ${ }^{22}$ as well as clinical effectiveness with both subjective and objective improvement in clinical signs and symptoms of OSD.24

The biofilm plays an important role in ocular surface pathology and homeostasis, as evidenced by its age-old, nuanced complexity and ability to occur around mucous membrane junctions of the eye. It is part of the reason why, as we age, we are more likely to encounter chronic, recalcitrant OSD. Furthermore, it explains why many therapies fail when treating these common conditions; they simply do not reach their intended target. It is the hearty and self-propagating extracellular matrix that protects these phenotypically diverse microorganisms. The single planktonic bacterium is much less fortunate, much less protected and much more susceptible to conventional antibiotic therapy. We hope that some of the newer therapeutic agents currently under investigation have a definitive role in the care of our patients. The evidence is encouraging and suggests that novel antibiofilm therapies will be coming to ophthalmology clinics in the near future. $]$
1. Lemp MA, Nichols KK. Blepharitis in the United States 2009: a survey-based perspective on prevalence and treatment. OCu Surf. 2009:7(2 Suppl.):S1-S14.

2. Paulsen AJ, Cruickshanks KJ, Fischer ME, et al. Dry eye in the beaver dam offspring study: prevalence, risk factors, and health-related quality of life. Am J Ophthalmol. 2014:157:799806.

3. Craig JP, Nichols KK, Akpek EK, et al. TFOS DEWS II definition and classification report. Ocul Surf. 2017;15:276-83.

4. Milner MS, Beckman KA, Luchs Jl, et al. Dysfunctional tear syndrome: dry eye disease and associated tear film disorders - new strategies for diagnosis and treatment. Curr Opin Ophthalmol. 2017; 28(Suppl. 1):3-47.

5. Donnenfeld ED, Holland EJ. Consider biofilm as alternative theory of dry eye etiology. Haelio Ocular Surgery News. 2019; (September 10). Available at: www.healio.com/news/ ophthalmology/20190822/consider-biolm-as-alternative-theoryof-dry-eye-etiology (accessed 5 July 2021).
6. Rynerson JM, Perry HD. DEBS - a unification theory for dry eye and blepharitis. Clin Ophthalmol. 2016;10:2455-67.

7. Gunn IS Bakaletz LO Wozniak DJ. What's on the outside matters: the role of the extracellular polymeric substance of Gram-negative biofilms in evading host immunity and as a target for therapeutic intervention. J Biol Chem. as a target for therap $291: 12538-46$

8. Kostakioti M, Hadjifrangiskou M, Hultgren SJ. Bacterial biofilms: development, dispersal, and therapeutic strategies in the dawn of the postantibiotic era. Cold Spring Harb Perspect Med. 2013;3:a010306.

9. Gomes LC, Mergulhão FJ. SEM analysis of surface impact on biofilm antibiotic treatment. Scanning. 2017;2017:2960194.

10. McDermott A. Antimicrobial compounds in tears. Exp Eye Res. 2013;117:53-61.

11. Grzybowski A, Brona P, Kim SJ. Microbial flora and resistance in ophthalmology: a review. Graefes Arch Clin Exp Ophthalmol. 2017;255:851-62.
12. Dong Q, Brulc JM, lovieno A, et al. Diversity of bacteria at healthy human conjunctiva. Invest Ophthalmol Vis Sci. 2011;52:5408-13.

13. Elder MJ, Stapleton F, Evans E, Dart JK. Biofilm-related infections in ophthalmology. Eye (Lond). 1995;9(Pt 1):102-9.

14. Bispo PJ, Haas W, Gilmore MS. Biofilms in infections of the eye Pathogens. 2015;4:111-36.

15. Hinojosa J, Patel N, Zhu M, et al. Antimicrobial efficacy of contact lens care solutions against neutrophil-enhanced bacterial biofilms. Trans/ Vis Sci Technol. 2017;6:11.

16. Wu EC, Kowalski RP, Romanowski EG, et al. AzaSite ${ }^{\circledR}$ inhibits Staphylococcus aureus and coagulase-negative Staphylococcus biofilm formation in vitro. J Ocul Pharmacol Ther. 2010;26:557-62.

17. Aslam S. Effect of antibacterials on biofilms. Am I Infect Control 2008;36:S175.

18. Fey PD, Olson ME. Current concepts in biofilm formation of Staphylococcus epidermidis. Future Microbiol. 2010;5:917-33. 
19. Archer NK, Mazaitis MJ, Costerton JW, et al. Staphylococcus aureus biofilms: properties, regulation, and roles in human disease. Virulence. 2011;2:445-59.

20. Macià MD, Rojo-Molinero E, Oliver A. Antimicrobial

susceptibility testing in biofilm-growing bacteria. Clin Microbiol Susceptibility testing in

21. Chen CJ, Chen CC, Ding SJ. Effectiveness of hypochlorous acid to reduce the biofilms on titanium alloy surfaces in vitro. Int $J$ Mol Sci. 2016;17:1161.

22. Tessema B, Wycherly B, Arumugam S, et al. Efficacy of dilute povidone-iodine preparations against multi-drug resistant biofilms of Staphylococcus aureus, Klebsiella pneumoniae, Pseudomonas aeruginosa and Candida albicans. Paripex Indian J Res. 2018;7:376-7.
23. Korb DR, Blackie CA. Debridement-scaling: a new procedure that increases meibomian gland function and reduces dry eye symptoms. Cornea. 2013;32:1554-7.

24. Pelletier JS, Capriotti KD, Stewart KS, et al. A novel transdermal ophthalmic preparation for blepharitis in a dilute povidoneophthalmic preparation for blepharitis in a dilute povidone-
iodine and dimethyl sulfoxide (DMSO) system: a case series. EC ophthalmol. 2018;9:129-34. 